\title{
RIGGED NON-TANGENTIAL MAXIMAL FUNCTION ASSOCIATED WITH TOEPLITZ OPERATORS AND HANKEL OPERATORS
}

\author{
JiNGBO XIA
}

\begin{abstract}
We give a sufficient condition for the boundedness of products of Toeplitz operators and Hankel operators in terms of a distributional inequality for the symbol functions.
\end{abstract}

Let $T$ denote the unit circle and $d m$ the normalized Lebesgue measure on $T$. For $1 \leq p \leq \infty, L^{p}$ stands for $L^{p}(T, d m)$. As usual, $H^{p}$ is the Hardy subspace of $L^{p}$. Let $P: L^{2} \rightarrow H^{2}$ be the orthogonal projection. For $f \in L^{2}$, the Toeplitz operator $T_{f}$ and the Hankel operator $H_{f}$ are defined by the formulas $T_{f} \varphi=P f \varphi$ and $H_{f} \varphi=(1-P) f \varphi, \varphi \in H^{2}$, whenever these expressions make sense. Thus the domains of $T_{f}$ and $H_{f}$ contain at least $H^{\infty}$.

Let $D$ be the open disc $\{z \in \mathbf{C}:|z|<1\}$. We denote the Poisson kernel associated with $z \in D$ by $P_{z}$. That is, $P_{z}(\tau)=\left(1-|z|^{2}\right) /|1-\bar{\tau} z|^{2}$. We write $f(z)=\int_{T} f P_{z} d m, z \in D$, for $f \in L^{1}$. In particular, for any measurable set $E \subset T, \chi_{E}(z)=\int_{E} P_{z} d m$ is the value of the harmonic extension of $\chi_{E}$ at $z$. The well-known probabilistic interpretation of $\chi_{E}(z)$ may help put the results stated below in perspective: $\chi_{E}(z)$ is the probability that a Brownian walker starting at the point $z$ will exit $D$ through $E$.

In this note we will address a question raised by Sarason in $[\mathbf{5}, \mathbf{6}]$, namely when is the product $T_{g} T_{f}$ a bounded operator on $H^{2}$ ? This problem, which is non-trivial only if at least one symbol function is unbounded, arose from Sarason's study of exposed points in the unit ball of $H^{1}$. He showed that, for outer functions $f, g \in H^{2}$, a necessary condition for $T_{g} T_{\bar{f}}$ to be bounded is that

$$
\sup _{|z|<1} \int_{T}|g|^{2} P_{z} d m \int_{T}|f|^{2} P_{z} d m<\infty .
$$

An argument due to Treil (see [6]) further shows that this condition is necessary for the boundedness of $T_{g} T_{\bar{f}}$ whenever $f, g \in H^{2}$. Sarason observed that a related (but strictly more general) problem is the boundedness of the product of Hankel operators $H_{g}^{*} H_{f}$.

Using the ideas of Axler, Chang and Sarason [1], mainly the area-integral function technique, Zheng showed in [10] that for $f, g \in L^{2}$, if there is an 
$\epsilon>0$ such that

$$
N_{1}=\sup _{|z|<1} \int_{T}|f-f(z)|^{2+\epsilon} P_{z} d m \int_{T}|g-g(z)|^{2+\epsilon} P_{z} d m<\infty,
$$

then $H_{g}^{*} H_{f}$ is bounded. He also showed that for $f, g \in H^{2}$, if there is an $\epsilon>0$ such that

$$
N_{2}=\sup _{|z|<1} \int_{T}|f|^{2+\epsilon} P_{z} d m \int_{T}|g|^{2+\epsilon} P_{z} d m<\infty,
$$

then $T_{g} T_{\bar{f}}$ is bounded. Recall that the theorem of Marcinkiewicz and Zygmund [4] asserts that Lusin's area-integral operator $S$ is bounded on $H^{p}$ for all $0<p<\infty$. Because of the properties of the Hilbert transform, it follows immediately that $S$ is bounded on $L^{p}$ when $p>1$. This is the key fact on which [10] relied.

This result was generalized by Treil, Volberg and Zheng to the setting of Orlicz spaces and Lorentz spaces [9]. In both [10] and [9], Hölder's inequality was used in an essential way in their estimates for the boundedness of the operators in question. It is well known that estimates relying on Hölder's inequality are usually less than optimal because of the nature of that inequality. In this paper we show that there are ways to get around Hölder's inequality, thereby obtaining sharper results.

The starting point of our investigation is Stein's result that $S$ also has weak-type $(1,1)[7]$. This suggests to us that the area-integral technique in $[\mathbf{1}, \mathbf{9}, \mathbf{1 0}]$ can be further exploited. Note that, by Hölder's inequality, (1) and (2) respectively imply that for any $z \in D$ and for any measurable sets $A, B \subset T$,

$$
\begin{gathered}
\int_{A}|f-f(z)| P_{z} d m \int_{B}|g-g(z)| P_{z} d m \leq N_{1}^{1 /(2+\epsilon)}\left(\chi_{A}(z) \chi_{B}(z)\right)^{(1+\epsilon) /(2+\epsilon)} \\
\int_{A}|f| P_{z} d m \int_{B}|g| P_{z} d m \leq N_{2}^{1 /(2+\epsilon)}\left(\chi_{A}(z) \chi_{B}(z)\right)^{(1+\epsilon) /(2+\epsilon)}
\end{gathered}
$$

We will replace the factor $\left(\chi_{A}(z) \chi_{B}(z)\right)^{(1+\epsilon) /(2+\epsilon)}$ in the above by something much larger and still obtain boundedness for $H_{g}^{*} H_{f}$ and $T_{g} T_{\bar{f}}$.

To state our main results, we need to introduce a class of weight functions. Let $\mathcal{W}$ denote the collection of functions $w:(0,1] \rightarrow(0,1]$ which are nondecreasing and have the property that

$$
\int_{0}^{1}\left(\frac{w(t)}{t^{2}}\right)^{2 / 3} d t<\infty
$$


Theorem 1. Suppose that $f, g \in L^{2}$ have the following property: There are $u, v \in \mathcal{W}$ and a positive number $N$ such that for any $z \in D$ and for any measurable sets $A, B \subset T$ of positive measure,

$$
\int_{A}|f-f(z)| P_{z} d m \int_{B}|g-g(z)| P_{z} d m \leq N u\left(\chi_{A}(z)\right) v\left(\chi_{B}(z)\right) .
$$

Then $H_{g}^{*} H_{f}$ is bounded.

Theorem 2. Suppose that $f, g \in L^{2}$ have the following property: There are $u, v \in \mathcal{W}$ and a positive number $N$ such that for any $z \in D$ and for any measurable sets $A, B \subset T$ of positive measure,

$$
\int_{A}|f| P_{z} d m \int_{B}|g| P_{z} d m \leq N u\left(\chi_{A}(z)\right) v\left(\chi_{B}(z)\right) .
$$

Then $T_{g} T_{f}$ is bounded.

The above-mentioned boundedness results of Zheng are recovered by applying these theorems to the case where $u(t)=v(t)=t^{(1+\epsilon) /(2+\epsilon)}=t^{1 / 2}$. $t^{\epsilon /(4+2 \epsilon)}$. What original motivated our investigation were examples such as $u(t)=v(t)=t^{1 / 2} \cdot(1-\log t)^{-(3+\epsilon) / 2}, \epsilon>0$.

Our main technical innovation is the introduction of a rigged non-tangential maximal function. Section 1 contains an $L^{1}$-boundedness result for this maximal function. The proofs of Theorems 1 and 2 are given in Section 2 after further preparations.

\section{Rigged non-tangential maximal function.}

For each $\tau \in T$, let $\Gamma_{\tau}=\{z:|\tau-z|<2(1-|z|), 3 / 4<|z|<1\}$. Suppose that $F, G$ are continuous maps from $D$ into $L^{2}$ with respect to the norm topology. To avoid confusion with complex-valued functions we denote their values at $z \in D$ by $F_{z}$ and $G_{z}$ respectively. In other words, for each $z \in D$, $F_{z}$ and $G_{z}$ are themselves functions on $T$. Given $\varphi, \psi \in L^{2}$, we introduce the rigged non-tangential maximal function

$$
M_{F, G}(\varphi, \psi)(\tau)=\sup _{z \in \Gamma_{\tau}} \int_{T}\left|\varphi F_{z}\right| P_{z} d m \int_{T}\left|\psi G_{z}\right| P_{z} d m, \quad \tau \in T .
$$

To simplify notation, the Lebesgue measure of a measurable set $E \subset T$ will be denoted by $|E|$. Also, for a real-valued function $f$ on $T$ and a $\lambda \in$ $\mathbf{R}$, the set $\{\tau \in T: f(\tau)>\lambda\}$ will simply be denoted by $\{f>\lambda\}$. The sets $\{f \leq \lambda\},\left\{\lambda_{1} \leq f<\lambda_{2}\right\}$, etc., are accordingly understood.

For each $\varphi \in L^{1}$, denote its usual non-tagential maximal function by $M_{\mathrm{nt}}(\varphi)$. That is,

$$
M_{\mathrm{nt}}(\varphi)(\tau)=\sup _{z \in \Gamma_{\tau}}|\varphi(z)|, \quad \tau \in T
$$


Recall that there is an absolute constant $C>0$ such that $M_{\mathrm{nt}}(\varphi) \leq C M(\varphi)$, where $M(\varphi)$ is the Hardy-Littlewood maximal function of $\varphi$ [3, page 24]. This means $M_{\text {nt }}$ is of weak-type $(1,1)$. In other words, there is an absolute constant $C_{\text {nt }}>0$ such that $\left|\left\{M_{\mathrm{nt}}(\varphi)>\lambda\right\}\right| \leq C_{\mathrm{nt}}\|\varphi\|_{1} / \lambda$ for all $\varphi \in L^{1}$ and $\lambda>0$. In particular, if $E$ is a measurable set in $T$, then

$$
\left|\left\{M_{\mathrm{nt}}\left(\chi_{E}\right)>2^{-i}\right\}\right| \leq C_{\mathrm{nt}} 2^{i}|E|, \quad i \in \mathbf{N} .
$$

Proposition 1.1. Suppose that $F, G$ are continuous maps from $D$ into $L^{2}$ which have the following property: There exist an $N>0$ and $u, v \in \mathcal{W}$ such that for any $z \in D$ and any measurable sets $A, B \subset T$ with $|A|>0$ and $|B|>0$,

$$
\int_{A}\left|F_{z}\right| P_{z} d m \int_{B}\left|G_{z}\right| P_{z} d m \leq N u\left(\chi_{A}(z)\right) v\left(\chi_{B}(z)\right) .
$$

Then there is a $K>0$ such that for any $\varphi, \psi \in L^{2}$,

$$
\left\|M_{F, G}(\varphi, \psi)\right\|_{1} \leq K\|\varphi\|_{2}\|\psi\|_{2}
$$

Proof. Without loss of generality, we may assume that $u(1)=v(1)=1$. Set $u_{i}=2^{i / 3}\left(u\left(2^{-i}\right)\right)^{2 / 3}$ and $v_{i}=2^{i / 3}\left(v\left(2^{-i}\right)\right)^{2 / 3}, i \in \mathbf{Z}_{+}$. It follows from the monotonicity of $u$ and $v$ that

$$
\begin{aligned}
U & =\sum_{i=0}^{\infty} u_{i} \leq 1+\frac{2^{1 / 3}}{\log 2} \sum_{i=0}^{\infty} \int_{2^{-i-1}}^{2^{-i}}\left(u^{2}(t) / t\right)^{1 / 3} \frac{d t}{t} \\
& =1+\frac{2^{1 / 3}}{\log 2} \int_{0}^{1}\left(\frac{u(t)}{t^{2}}\right)^{2 / 3} d t<\infty, \\
V & =\sum_{i=0}^{\infty} v_{i} \leq 1+\frac{2^{1 / 3}}{\log 2} \sum_{i=0}^{\infty} \int_{2^{-i-1}}^{2^{-i}}\left(v^{2}(t) / t\right)^{1 / 3} \frac{d t}{t} \\
& =1+\frac{2^{1 / 3}}{\log 2} \int_{0}^{1}\left(\frac{v(t)}{t^{2}}\right)^{2 / 3} d t<\infty .
\end{aligned}
$$

It suffices to consider non-negative $\varphi, \psi \in L^{2}$ with $\|\varphi\|_{2}=\|\psi\|_{2}=1$. Define

$$
\begin{aligned}
& A_{k, 0}=\left\{\varphi^{2} \leq 2^{k}\right\}, \quad B_{k, 0}=\left\{\psi^{2} \leq 2^{k}\right\}, \\
& A_{k, i}=\left\{2^{k+i-1} / u_{i-1} \leq \varphi^{2}<2^{k+i} / u_{i}\right\}, \\
& B_{k, i}=\left\{2^{k+i-1} / v_{i-1} \leq \psi^{2}<2^{k+i} / v_{i}\right\},
\end{aligned}
$$

$k, i \in \mathbf{N}$. (Recall that $u$ and $v$ do not vanish on $(0,1]$.) For such a pair of $k$ and $i$, let

$$
X_{k, i}=\left\{M_{\mathrm{nt}}\left(\chi_{A_{k, i}}\right)>2^{-i}\right\}, \quad Y_{k, i}=\left\{M_{\mathrm{nt}}\left(\chi_{B_{k, i}}\right)>2^{-i}\right\} .
$$


It follows from (1.2) that

$$
\begin{aligned}
& \int_{T}\left|F_{z} \varphi\right| P_{z} d m \int_{T}\left|G_{z} \psi\right| P_{z} d m \\
& \leq \sum_{i, j=0}^{\infty}\left(\frac{2^{2 k+i+j}}{u_{i} v_{j}}\right)^{1 / 2} \int_{A_{k, i}}\left|F_{z}\right| P_{z} d m \int_{B_{k, j}}\left|G_{z}\right| P_{z} d m \\
& \leq N 2^{k} \sum_{i, j=0}^{\infty}\left(\frac{2^{i+j}}{u_{i} v_{j}}\right)^{1 / 2} u\left(\chi_{A_{k, i}}(z)\right) v\left(\chi_{B_{k, j}}(z)\right) \\
& =N 2^{k} \sum_{i=0}^{\infty} \sqrt{\frac{2^{i}}{u_{i}}} u\left(\chi_{A_{k, i}}(z)\right) \sum_{j=0}^{\infty} \sqrt{\frac{2^{j}}{v_{j}}} v\left(\chi_{B_{k, j}}(z)\right) .
\end{aligned}
$$

Now if $\tau_{0} \in T \backslash \cup_{i=1}^{\infty}\left(X_{k, i} \cup Y_{k, i}\right)$, then $\chi_{A_{k, i}}(z) \leq 2^{-i}$ and $\chi_{B_{k, j}}(z) \leq 2^{-j}$ for any $z \in \Gamma_{\tau_{0}}$ and $i, j \in \mathbf{N}$. The monotonicity of $u$ then implies that $u_{i}^{3 / 2}=2^{i / 2} u\left(2^{-i}\right) \geq 2^{i / 2} u\left(\chi_{A_{k, i}}(z)\right)$. That is, $\left(2^{i} / u_{i}\right)^{1 / 2} u\left(\chi_{A_{k, i}}(z)\right) \leq u_{i}$. Similarly $\left(2^{j} / v_{j}\right)^{1 / 2} v\left(\chi_{A_{k, j}}(z)\right) \leq v_{j}$. Therefore it follows from (1.3) that

$$
\int_{T}\left|F_{z} \varphi\right| P_{z} d m \int_{T}\left|G_{z} \psi\right| P_{z} d m \leq N U V 2^{k}
$$

if $\tau_{0} \in T \backslash \cup_{i=1}^{\infty}\left(X_{k, i} \cup Y_{k, i}\right)$ and $z \in \Gamma_{\tau_{0}}$. This implies that

$$
\left\{M_{F, G}(\varphi, \psi)>N U V 2^{k}\right\} \subset \bigcup_{i=1}^{\infty}\left(X_{k, i} \cup Y_{k, i}\right)
$$

By (1.1), $\left|X_{k, i}\right| \leq C_{\mathrm{nt}} 2^{i}\left|A_{k, i}\right|,\left|Y_{k, i}\right| \leq C_{\mathrm{nt}} 2^{i}\left|B_{k, i}\right|$. Thus

$$
\left|\left\{M_{F, G}(\varphi, \psi) / N U V>2^{k}\right\}\right| \leq C_{\mathrm{nt}} \sum_{i=1}^{\infty} 2^{i}\left(\left|A_{k, i}\right|+\left|B_{k, i}\right|\right)
$$

Since $\|f\|_{1} \leq 2|T|+\sum_{k=1}^{\infty} 2^{k+1}\left|\left\{2^{k}<|f| \leq 2^{k+1}\right\}\right|$ for every $f \in L^{1}$, we have

$$
\left\|M_{F, G}(\varphi, \psi) / N U V\right\|_{1} \leq 2+2 C_{\mathrm{nt}} \sum_{k=1}^{\infty} \sum_{i=1}^{\infty} 2^{k+i}\left(\left|A_{k, i}\right|+\left|B_{k, i}\right|\right) .
$$


Because $u \leq 1$ on $(0,1], 2^{k+i-1} / u_{i-1} \geq 2^{2 i / 3} \geq 1$ for all $k, i \in \mathbf{N}$. Therefore

$$
\begin{aligned}
& \sum_{k, i=1}^{\infty} 2^{k+i}\left|A_{k, i}\right| \\
& \leq 2 \sum_{k, i=1}^{\infty} 2^{k+i-1}\left|\left\{\varphi^{2} \geq 2^{k+i-1} / u_{i-1}\right\}\right| \\
& =2 \sum_{\ell=0}^{\infty} \sum_{2^{\ell} \leq 2^{k+i-1} / u_{i-1}<2^{\ell+1}}\left(2^{k+i-1} / u_{i-1}\right) u_{i-1}\left|\left\{\varphi^{2} \geq 2^{k+i-1} / u_{i-1}\right\}\right| \\
& \leq 2 \sum_{\ell=0}^{\infty} 2^{\ell+1}\left|\left\{\varphi^{2} \geq 2^{\ell}\right\}\right| \sum_{2^{\ell \leq 2^{k+i-1} / u_{i-1}<2^{\ell+1}}} u_{i-1} .
\end{aligned}
$$

Note that for each pair of $i \in \mathbf{N}$ and $\ell \in \mathbf{Z}_{+}$, there is at most one $k \in \mathbf{N}$ for which the inequality $2^{\ell} \leq 2^{k+i-1} / u_{i-1}<2^{\ell+1}$ holds. This means that for each fixed $\ell$,

$$
\sum_{2^{\ell} \leq 2^{k+i-1} / u_{i-1}<2^{\ell+1}} u_{i-1} \leq \sum_{i=1}^{\infty} u_{i-1}=U .
$$

Also, since $1+2+\cdots+2^{\ell}<2^{\ell+1}, \sum_{\ell=0}^{\infty} 2^{\ell}\left|\left\{\varphi^{2} \geq 2^{\ell}\right\}\right| \leq 2\left\|\varphi^{2}\right\|_{1}=2\|\varphi\|_{2}^{2}=2$. Hence it follows from (1.5) that

$$
\sum_{k, i=1}^{\infty} 2^{k+i}\left|A_{k, i}\right| \leq 8 U
$$

By the same argument,

$$
\sum_{k, i=1}^{\infty} 2^{k+i}\left|B_{k, i}\right| \leq 8 V .
$$

Combining these with (1.4), we see that

$$
\left\|M_{F, G}(\varphi, \psi)\right\|_{1} \leq N U V\left(2+16 C_{\mathrm{nt}}(U+V)\right)
$$

This completes the proof.

\section{Area-Integral.}

For each $z=|z| e^{i \theta_{z}} \in D$, define the open arcs

$$
I_{z}=\left\{e^{i \theta}:\left|\theta-\theta_{z}\right|<(1-|z|) / 2\right\}, \quad J_{z}=\left\{e^{i \theta}:\left|\theta-\theta_{z}\right|<3(1-|z|) / 2\right\} .
$$


Because of the normalization $|T|=1$, we have $\left|I_{z}\right|=(1-|z|) / 2 \pi$. If $\tau=e^{i \theta}$ is a point in $I_{z}$, then $\tau-z=e^{i \theta}(1-|z|)+z\left(e^{i\left(\theta-\theta_{z}\right)}-1\right)$. Therefore $|\tau-z| \leq$ $(1-|z|)+(e-1)\left|\theta-\theta_{z}\right|<2(1-|z|)$. That is, if $3 / 4<|z|<1$ and $\tau \in I_{z}$, then $z \in \Gamma_{\tau}$.

Let $d A(z)$ denote the area measure on $D$. Recall that for each $\varphi \in L^{1}$, Lusin's area-integral function is defined by the formula

$$
S(\varphi)(\tau)=\left(\int_{\Gamma_{\tau}}|\nabla \varphi(z)|^{2} d A(z)\right)^{1 / 2} .
$$

Using the Calderón-Zygmund decomposition of $L^{1}$-functions, Stein proved that $S$ is of weak-type $(1,1)$ [7, Lemma 12]. (Stein's proof was given for half-spaces in $\mathbf{R}^{n}$, but, with obvious modifications, the proof works in $D$ as well.) That is, there is an absolute constant $C_{S}>0$ such that

$$
|\{S(\xi)>\lambda\}| \leq C_{S}\|\xi\|_{1} / \lambda
$$

for all $\xi \in L^{1}$ and $\lambda>0$.

For each $0<a<1$ and each $\tau \in T$, let $\Gamma_{\tau, a}=\Gamma_{\tau} \cap\{z: 1-a<|z|<1\}$. We set $\Gamma_{\tau, 0}=\emptyset$. Define the truncated area-integral function

$$
S_{a}(\varphi)(\tau)=\left(\int_{\Gamma_{\tau, a}}|\nabla \varphi(z)|^{2} d A(z)\right)^{1 / 2} .
$$

Recall that $\partial=((\partial / \partial x)-i(\partial / \partial y)) / 2$ and $\bar{\partial}=((\partial / \partial x)+i(\partial / \partial y)) / 2$ in real variables. Thus $|\nabla \varphi(z)|^{2}=2\left(|\partial \varphi(z)|^{2}+|\bar{\partial} \varphi(z)|^{2}\right)$. For $\varphi \in L^{2}$, we have $|\nabla((1-P) \varphi)(z)|^{2}=2|\bar{\partial}((1-P) \varphi)(z)|^{2}=2|\bar{\partial} \varphi(z)|^{2} \leq|\nabla \varphi(z)|^{2}$ and $|\nabla(P \varphi)(z)|^{2}=2|\partial(P \varphi)(z)|^{2}=2|\partial \varphi(z)|^{2} \leq|\nabla \varphi(z)|^{2}$. Hence for any given $\varphi \in L^{2}$,

$$
S_{a}((1-P) \varphi) \leq S_{a}(\varphi), \quad S_{a}(P \varphi) \leq S_{a}(\varphi) .
$$

There is a $C_{2.3}>0$ such that for any $f \in L^{1}, 3 / 4<|z|<1$ and $\tau \in I_{z}$,

$$
S_{2(1-|z|)}\left(\chi_{T \backslash J_{z}} f\right)(\tau) \leq C_{2.3} \int_{T}|f| P_{z} d m .
$$

To verify this elementary claim, note that

$$
\left|\bar{\partial}\left(\chi_{T \backslash J_{z}} f\right)(\zeta)\right|=\left|\frac{d}{d \bar{\zeta}} \int_{T \backslash J_{z}} \frac{\bar{\zeta} \gamma f(\gamma)}{1-\bar{\zeta} \gamma} d m(\gamma)\right| \leq \int_{T \backslash J_{z}} \frac{|f(\gamma)|}{|1-\zeta \bar{\gamma}|^{2}} d m(\gamma) .
$$

There is a $C_{1}>0$ such that $C_{1}|\gamma-\tau| \geq|\gamma-z|$ when $\tau \in I_{z}$ and $\gamma \in T \backslash J_{z}$. Also, there is a $C_{2}>0$ such that $C_{2} d\left(\gamma, \Gamma_{\tau}\right) \geq|\gamma-\tau| \geq|\gamma-z| / C_{1}$ for such $z, \tau, \gamma$. That is, for $\zeta \in \Gamma_{\tau}$ and $\gamma \in T \backslash J_{z}, C_{1} C_{2}|\gamma-\zeta| \geq|\gamma-z|$. Hence

$$
\left|\bar{\partial}\left(\chi_{T \backslash J_{z}} f\right)(\zeta)\right| \leq\left(C_{1} C_{2}\right)^{2} \int_{T \backslash J_{z}} \frac{|f(\gamma)|}{|1-z \bar{\gamma}|^{2}} d m(\gamma)
$$


for $\zeta \in \Gamma_{\tau}$. There is a $C_{3}>0$ such that the area of $\Gamma_{\tau, 2(1-|z|)}$ does not exceed $C_{3}\left(1-|z|^{2}\right)^{2}$. Squaring the above and integrating over $\Gamma_{\tau, 2(1-|z|)}$, we see that

$$
\int_{\Gamma_{\tau, 2(1-|z|)}}\left|\bar{\partial}\left(\chi_{T \backslash J_{z}} f\right)(\zeta)\right|^{2} d A(\zeta) \leq C_{3}\left(\left(C_{1} C_{2}\right)^{2} \int_{T}|f| P_{z} d m\right)^{2} .
$$

Repeating the above argument with $\partial$ in place of $\bar{\partial}$, we see $(2.3)$ holds with $C_{2.3}=2 C_{3}^{1 / 2}\left(C_{1} C_{2}\right)^{2}$.

Proposition 2.1. There is an absolute constant $B_{2.1}>0$ such that for any continuous maps $F, G: D \rightarrow L^{2}, \varphi, \psi \in L^{2}$, and any $3 / 4<|z|<1$,

$$
\left|\left\{S_{2(1-|z|)}\left(F_{z} \varphi\right) S_{2(1-|z|)}\left(G_{z} \psi\right) \leq B_{2.1} M_{F, G}(\varphi, \psi)\right\} \cap I_{z}\right| \geq\left|I_{z}\right| / 2 .
$$

Proof. Since $F_{z} \varphi=F_{z} \varphi \chi_{J_{z}}+F_{z} \varphi \chi_{T \backslash J_{z}}$, by the subadditivity of $S_{a}$ and (2.3),

$$
\begin{aligned}
\left\{S_{2(1-|z|)}\left(F_{z} \varphi\right)\right. & \left.>\frac{12 C_{S}}{\left|J_{z}\right|} \int_{J_{z}}\left|F_{z} \varphi\right| d m+2 C_{2.3} \int_{T}\left|F_{z} \varphi\right| P_{z} d m\right\} \cap I_{z} \\
& \subset\left\{S_{2(1-|z|)}\left(\chi_{J_{z}} F_{z} \varphi\right)>\frac{12 C_{S}}{\left|J_{z}\right|} \int_{J_{z}}\left|F_{z} \varphi\right| d m\right\} \cap I_{z} \\
& \subset\left\{S\left(\chi_{J_{z}} F_{z} \varphi\right)>\frac{12 C_{S}}{\left|J_{z}\right|} \int_{J_{z}}\left|F_{z} \varphi\right| d m\right\} \cap I_{z} .
\end{aligned}
$$

If $\left\|\chi_{J_{z}} F_{z} \varphi\right\|_{1}=0$, then the above set is empty. If $\left\|\chi_{J_{z}} F_{z} \varphi\right\|_{1} \neq 0$, apply (2.1) to the case where $\xi=\chi_{J_{z}} F_{z} \varphi$ and $\lambda=12 C_{S} \int_{J_{z}}\left|F_{z} \varphi\right| d m /\left|J_{z}\right|=$ $12 C_{S}\|\xi\|_{1} / 3\left|I_{z}\right|$. Therefore we have

$$
\begin{aligned}
\mid\left\{S_{2(1-|z|)}\left(F_{z} \varphi\right)\right. & \left.>\frac{12 C_{S}}{\left|J_{z}\right|} \int_{J_{z}}\left|F_{z} \varphi\right| d m+2 C_{2.3} \int_{T}\left|F_{z} \varphi\right| P_{z} d m\right\} \cap I_{z} \mid \\
& \leq\left|\left\{S\left(\chi_{J_{z}} F_{z} \varphi\right)>\frac{12 C_{S}}{\left|J_{z}\right|} \int_{J_{z}}\left|F_{z} \varphi\right| d m\right\}\right| \leq\left|I_{z}\right| / 4
\end{aligned}
$$

in any case. There is a $C_{4}>0$ such that $\chi_{J_{z}} /\left|J_{z}\right| \leq C_{4} P_{z}$. Hence

$$
\left|\left\{S_{2(1-|z|)}\left(F_{z} \varphi\right)>\left(12 C_{4} C_{S}+2 C_{2.3}\right) \int_{T}\left|F_{z} \varphi\right| P_{z} d m\right\} \cap I_{z}\right| \leq\left|I_{z}\right| / 4 \text {. }
$$

Applying the same argument with $G_{z}, \psi$ in place of $F_{z}, \varphi$, we obtain

$$
\left|\left\{S_{2(1-|z|)}\left(G_{z} \psi\right)>\left(12 C_{4} C_{S}+2 C_{2.3}\right) \int_{T}\left|G_{z} \psi\right| P_{z} d m\right\} \cap I_{z}\right| \leq\left|I_{z}\right| / 4 .
$$

Let $B_{2.1}=\left(12 C_{4} C_{S}+2 C_{2.3}\right)^{2}$. Then the set $E$ which consists of all $\tau \in I_{z}$ such that

$$
S_{2(1-|z|)}\left(F_{z} \varphi\right)(\tau) S_{2(1-|z|)}\left(G_{z} \psi\right)(\tau) \leq B_{2.1} \int_{T}\left|F_{z} \varphi\right| P_{z} d m \int_{T}\left|G_{z} \psi\right| P_{z} d m
$$


has measure at least $\left|I_{z}\right| / 2$. Again, $z \in \Gamma_{\tau}$ if $\tau \in I_{z}$. Hence the inequality

$$
S_{2(1-|z|)}\left(F_{z} \varphi\right)(\tau) S_{2(1-|z|)}\left(G_{z} \psi\right)(\tau) \leq B_{2.1} M_{F, G}(\varphi, \psi)(\tau)
$$

holds whenever $\tau \in E$. This completes the proof.

It should be acknowledged that the idea of decomposing $F_{z} \varphi$ as $F_{z} \varphi \chi_{J_{z}}+$ $F_{z} \varphi \chi_{T \backslash J_{z}}$ in the above proof can be traced back to [1].

Proposition 2.2. There is an absolute constant $C_{2.2}>0$ such that the following hold true:

(i) For any $f, g \in L^{2}$, there is a $C(f, g)>0$ such that for any $\varphi, \psi \in H^{2}$ with the property that $H_{f} \varphi, H_{g} \psi \in L^{2}$, we have

$$
\left|\left\langle H_{f} \varphi, H_{g} \psi\right\rangle\right| \leq C_{2.2} \int_{T} M_{F, G}(\varphi, \psi) d m+C(f, g)\|\varphi\|_{2}\|\psi\|_{2},
$$

where $F, G: D \rightarrow L^{2}$ are defined by the formulas $F_{z}=f-f(z)$ and $G_{z}=g-g(z)$.

(ii) For any $f, g \in L^{2}$, there is a $B(f, g)>0$ such that for any $\varphi, \psi \in H^{2}$ with the property that $T_{f} \varphi, T_{g} \psi \in L^{2}$, we have

$$
\left|\left\langle T_{f} \varphi, T_{g} \psi\right\rangle\right| \leq C_{2.2} \int_{T} M_{F^{0}, G^{0}}(\varphi, \psi) d m+B(f, g)\|\varphi\|_{2}\|\psi\|_{2},
$$

where $F^{0}, G^{0}: D \rightarrow L^{2}$ are defined by the formulas $F_{z}^{0}=f$ and $G_{z}^{0}=g$.

Proof. (i) The harmonic extensions of $H_{f} \varphi$ and $H_{g} \psi$ vanish at 0 . Hence it follows from the Littlewood-Paley formula that

$$
\begin{aligned}
\left|\left\langle H_{f} \varphi, H_{g} \psi\right\rangle\right| & \leq \frac{1}{\pi} \int_{7 / 8<|z|<1}\left|\left\langle\nabla\left(H_{f} \varphi\right)(z), \nabla\left(H_{g} \psi\right)(z)\right\rangle_{\mathbf{C}^{2}}\right| \log \frac{1}{|z|} d A(z) \\
& +\frac{1}{\pi} \int_{|z| \leq 7 / 8}\left|\left\langle\nabla\left(H_{f} \varphi\right)(z), \nabla\left(H_{g} \psi\right)(z)\right\rangle_{\mathbf{C}^{2}}\right| \log \frac{1}{|z|} d A(z) .
\end{aligned}
$$

It is elementary that there is a $C(f, g)>0$ such that the second term above is bounded by $C(f, g)\|\varphi\|_{2}\|\psi\|_{2}$. Thus it suffices to estimate the first term.

Mimicking the definition of $\rho(w)$ on page 494 of [10], for each $\tau \in T$, let $a(\tau)$ be the largest $a \in[0,1 / 4]$ such that

$$
S_{a}\left(H_{f} \varphi\right)(\tau) S_{a}\left(H_{g} \psi\right)(\tau) \leq B_{2.1} M_{F, G}(\varphi, \psi)(\tau) .
$$

(We set $S_{0}(\xi)=0$.) We claim that the function

$$
\tau \mapsto S_{a(\tau)}\left(H_{f} \varphi\right)(\tau) S_{a(\tau)}\left(H_{g} \psi\right)(\tau)
$$


is measurable ${ }^{1}$ on $T$. In fact, for each fixed $\tau \in\left\{S\left(H_{f} \varphi\right)<\infty\right\} \cap\left\{S\left(H_{g} \psi\right)<\right.$ $\infty$, since the function $a \mapsto S_{a}\left(H_{f} \varphi\right)(\tau) S_{a}\left(H_{g} \psi\right)(\tau)$ is non-decreasing and continuous on $[0,1 / 4]$, we have

$$
\begin{aligned}
S_{a(\tau)}\left(H_{f} \varphi\right)(\tau) & S_{a(\tau)}\left(H_{g} \psi\right)(\tau) \\
& =\sup _{n} \min \left\{B_{2.1} M_{f, g}(\varphi, \psi)(\tau), S_{a_{n}}\left(H_{f} \varphi\right)(\tau) S_{a_{n}}\left(H_{g} \psi\right)(\tau)\right\},
\end{aligned}
$$

where $\left\{a_{n}\right\}$ is any chosen sequence which is dense in $[0,1 / 4]$. And $\left\{S\left(H_{f} \varphi\right)=\right.$ $\infty\} \cup\left\{S\left(H_{g} \psi\right)=\infty\right\}$ is a null set. Similarly $\tau \mapsto a(\tau)$ is also measurable.

We claim that $\int_{I_{z}} \chi_{\Gamma_{\tau, a(\tau)}}(z) d m(\tau) \geq(1-|z|) / 4 \pi$ when $7 / 8<|z|<1$. Indeed if

$$
S_{2(1-|z|)}((f-f(z)) \varphi)(\gamma) S_{2(1-|z|)}((g-g(z)) \psi)(\gamma) \leq B_{2.1} M_{F, G}(\varphi, \psi)(\gamma)
$$

then, because $H_{f} \varphi=H_{f-f(z)} \varphi, H_{f} \varphi=H_{f-f(z)} \varphi$, and because of (2.2), we have

$$
S_{2(1-|z|)}\left(H_{f} \varphi\right)(\gamma) S_{2(1-|z|)}\left(H_{g} \psi\right)(\gamma) \leq B_{2.1} M_{F, G}(\varphi, \psi)(\gamma) .
$$

That is, if $\gamma \in I_{z}$ is such that (2.4) holds, then $a(\gamma) \geq 2(1-|z|)>1-|z|$, consequently $|z|>1-a(\gamma)$. This implies $z \in \Gamma_{\gamma, a(\gamma)}$ since $z \in \Gamma_{\gamma}$ whenever $\gamma \in I_{z}$. But Proposition 2.1 tells us that the set of $\gamma$ 's in $I_{z}$ for which (2.4) holds has measure at least $\left|I_{z}\right| / 2$. In other words, when $7 / 8<|z|<1$, the function $\tau \mapsto \chi_{\Gamma_{\tau, a(\tau)}}(z)$ equals 1 on a subset of $I_{z}$ with measure at least $\left|I_{z}\right| / 2=(1-|z|) / 4 \pi$. This verifies our claim. Borrowing an idea from [10], we have

$$
\begin{aligned}
& \frac{1}{\pi} \int_{\tau / 8<|z|<1}(1-|z|)\left|\left\langle\nabla\left(H_{f} \varphi\right)(z), \nabla\left(H_{g} \psi\right)(z)\right\rangle\right| d A(z) \\
& \leq 4 \int_{\tau / 8<|z|<1}\left(\int_{I_{z}} \chi_{\Gamma_{\tau, a(\tau)}}(z) d m(\tau)\right)\left|\left\langle\nabla\left(H_{f} \varphi\right)(z), \nabla\left(H_{g} \psi\right)(z)\right\rangle_{\mathbf{C}^{2}}\right| d A(z) \\
& =4 \int_{T} \int_{7 / 8<|z|<1} \chi_{\Gamma_{\tau, a(\tau)}}(z)\left|\left\langle\nabla\left(H_{f} \varphi\right)(z), \nabla\left(H_{g} \psi\right)(z)\right\rangle_{\mathbf{C}^{2}}\right| d A(z) d m(\tau) \\
& \leq 4 \int_{T} S_{a(\tau)}\left(H_{f} \varphi\right)(\tau) S_{a(\tau)}\left(H_{g} \psi\right)(\tau) d m(\tau) \leq 4 B_{2.1} \int_{T} M_{f, g}(\varphi, \psi) d m .
\end{aligned}
$$

But there is a $C_{2.2 .1}>0$ such that $\log (1 /|z|)<C_{2.2 .1}(1-|z|)$ whenever $7 / 8<|z|<1$. Thus $C_{2.2}=4 C_{2.2 .1} B_{2.1}$ satisfies our requirement.

(ii) Note that $\left\langle T_{f} \varphi, T_{g} \psi\right\rangle=\langle P f \varphi, g \psi\rangle=\langle P(f \varphi-(f \varphi)(0)), g \psi-(g \psi)(0)\rangle+$ garbage. Now there is a $C^{\prime}(f, g)>0$ such that $C^{\prime}(f, g)\|\varphi\|_{2}\|\psi\|_{2}$ dominates

\footnotetext{
${ }^{1}$ Note that a similar measurability issue was overlooked on page 494 of [10].
} 
the garbage term. Applying the Littlewood-Paley formula to the other term,

$$
\begin{aligned}
\langle P(f \varphi-(f \varphi)(0)), g \psi- & (g \psi)(0)\rangle \\
& =\frac{1}{\pi} \int_{D}\langle\nabla(P f \varphi)(z), \nabla(g \psi)(z)\rangle_{\mathrm{C}^{2}} \log \frac{1}{|z|} d A(z),
\end{aligned}
$$

the rest of the proof proceeds as in (i) with the only modification that $F_{z}^{0}=f$ and $G_{z}^{0}=g$ now replace $F_{z}=f-f(z)$ and $G_{z}=g-g(z)$ respectively.

With the foregoing preparation, we are now ready to prove our main results.

Proof of Theorem 1 (resp. 2). By Proposition 2.2(i) (resp. 2.2(ii)), it suffices to show that there is a $K>0$ such that $\left\|M_{F, G}(\varphi, \psi)\right\|_{1} \leq K\|\varphi\|_{2}\|\psi\|_{2}$ when $F_{z}=f-f(z)$ and $G_{z}=g-g(z)$ (resp. $F_{z}=f$ and $G_{z}=g$ ). But with this notation, the assumption of the theorem now reads

$$
\int_{A}\left|F_{z}\right| P_{z} d m \int_{B}\left|G_{z}\right| P_{z} d m \leq N u\left(\chi_{A}(z)\right) v\left(\chi_{B}(z)\right)
$$

Proposition 1.1 asserts that such a $K$ exists under this condition.

\section{References}

[1] S. Axler, S.-Y. Chang and D. Sarason, Products of Toeplitz operator, Integral Eq. Operator Theory, 1 (1978), 285-309.

[2] R. Douglas, Banach algebra techniques in operator theory, Academic Press, New York, 1972.

[3] J. Garnett, Bounded analytic functions, Academic Press, New York, 1981.

[4] J. Marcinkiewicz and A. Zygmund, A theorem of Lusin, Duke J. Math., 4 (1938), 473-485.

[5] D. Sarason, Exposed points in $H^{1}$, II, Operator Theory: Adv. Appl., 48 (1990), 333-347.

[6] _ Products of Toeplitz operators, Lecture Notes in Mathematics, 1573, Springer-Verlag, Berlin, 1994.

[7] E. Stein, On the functions of Littlewood-Paley, Lusin and Marcinkiewicz, Trans. Amer. Math. Soc., 88 (1958), 430-466.

[8] - Singular integrals and differentiablity properties of functions, Princeton University Press, Princeton, 1970.

[9] S. Treil, A. Vilberg and D. Zheng, Hilbert transform, Toeplitz operators and Hankel operators, and invariant $A_{\infty}$ weights, preprint. 
[10] D. Zheng, The distribution function inequality and products of Toeplitz operators and Hankel operators, J. Funct. Anal., 138 (1996), 477-501.

Received August 20, 1996. This research was supported in part by the National Science Foundation grant DMS-9400600.

State University of New York at Buffalo

BufFALO, NY 14214

E-mail address: jxia@acsu.buffalo.edu 